\title{
Spontaneous attention to primed and nonprimed inputs
}

\author{
IRENE S. SCHWARTING and WILLIAM A. JOHNSTON \\ University of Utah, Salt Lake City, Utah
}

\begin{abstract}
The literature is ambiguous with respect to whether attention is drawn spontaneously to expected or unexpected items in mixed arrays. Several studies from our own laboratory indicate that even though expected words are more localizable than unexpected words in unmixed four-word arrays, showing a baseline advantage for expected words, unexpected words are sometimes more localizable than their expected companions in mixed arrays, suggesting that unexpected words attract attention (see, e.g., Johnston \& Schwarting, 1996). By contrast, Dark, Vochatzer, and VanVoorhis (1996) observed that expected words were more reportable than their unexpected companions in mixed, two-word arrays. However, because the Dark et al. research did not include arrays in which both words were expected, it is not clear whether their findings reflect an attentional effect over and above a baseline advantage of expected words. The present study added some additional controls in order to assess this possibility. The superior reportability of expected words was even greater in mixed arrays than in unmixed arrays, suggesting that expected words in mixed arrays attract attention. Following Johnston and Hawley (1994), the conflicting effects of expectancy on spontaneous attention are taken as further evidence that the mind/brain system is biased simultaneously toward both what it most expects and what it least expects to perceive.
\end{abstract}

Johnston and Hawley (1994) reviewed an extensive literature in support of the idea that organisms are biased simultaneously toward both what they most expect and what they least expect to experience. These opposing biases serve the adaptive function of allowing organisms to become attuned to the stable, predictable features of their environments and still remain sensitive to novel, unpredictable features. The present paper addresses the general idea of opposing perceptual biases by examining the role of expectancy in spontaneous attention. Suppose that observers are instructed to perceive as much as they can of scenes that contain both expected and unexpected objects that are exposed so briefly that only one object can be correctly identified and localized. Which kind of object is most likely to "capture attention" spontaneously: expected objects or unexpected objects? In line with Johnston and Hawley's proposal, the extant literature provides indications of both attentional biases.

Expectancy for an item can be experimentally elevated in at least two ways, neither of which necessarily implies the phenomenological state of conscious anticipation: by repeating the item several times within the experimental context or by preceding it with an associative prime. In most of our research, expectancy has been manipulated via repetition. We have consistently found evidence for the

This research formed part of a thesis submitted by I.S.S. to the University of Utah in partial fulfillment of a master's degree in psychology. We thank Francis Friedrich and David Strayer, thesis committee members, for their guidance in this project. Correspondence should be addressed to W. A. Johnston, Department of Psychology, University of Utah, 390 S. 1530 E. Room 502, Salt Lake City, UT 84112 (e-mail: johnston@psych.utah.edu). attention-drawing power of unexpected items (see, e.g., Johnston, Hawley, Plewe, Elliott, \& DeWitt, 1990; Johnston \& Schwarting, 1996, 1997; Johnston, Schwarting, \& Hawley, 1996). In a typical study, four-word arrays are presented to observers for up to $200 \mathrm{msec}$ before being backward masked. Following each array, one of the words is given as a probe and observers indicate the location that it occupied in the array. This paradigm encourages observers to process the entire display, as there is no way of knowing ahead of time which item will be probed. In line with a voluminous literature on general facilitatory effects of expectancy, accuracy of localization is usually higher for arrays composed entirely of repeated words than for arrays composed of nonrepeated words. However, this facilitatory effect of word repetition is at least reduced, and often reversed, when a lone nonrepeated word appears with three repeated words. That is, under certain conditions (see, e.g., Johnston \& Schwarting, 1996), a nonrepeated singleton is actually more localizable than any of the repeated field words with which it is arrayed. We have referred to the relatively high localizability of nonrepeated singletons as novel popout and the relatively low localizability of the repeated words with which they appear as familiar sink-in. ${ }^{1}$

In another study in our laboratory, DeWitt (1994) observed the same pattern of effects when expectancy was manipulated via associative priming rather than repetition. Each four-word array was preceded by a prime word that was related to all, three, one, or none of the words in the display. Localization accuracy was higher for arrays containing all primed words than for arrays containing all nonprimed words, a baseline effect consistent with the well-established facilitatory effects of priming on per- 
ception. However, relative to these baselines, localization of nonprimed words improved and that of primed words declined in arrays containing a mixture of primed and nonprimed words. Thus, DeWitt observed nonprimed popout and primed sink-in, providing further evidence that attention is drawn spontaneously to unexpected items.

By contrast, in another study of spontaneous attention using a different experimental paradigm, Dark, Vochatzer, and Van Voorhis (1996) reported evidence that attention is drawn to expected inputs. Observers performed a whole-report task on 100-msec, two-word arrays. Each of the arrays of interest was preceded by a prime word and followed by a backward mask. The prime word was related to either just one (mixed arrays) or neither (unmixed, nonprimed arrays) of the two words in the succeeding array. Participants rarely could report more than one of the two words. Report accuracy was highest for primed words, lowest for their nonprimed companions, and between these levels for the nonprimed, baseline arrays, suggestive of primed popout and nonprimed sink-in.

Taken together, our own prior findings and those of Dark et al. (1996) appear to indicate conflicting attentional biases. However, although the design of the Dark et al. research was adequate for their purposes, the incorporation of additional controls would extend the generality of their findings and provide more convincing evidence of a perceptual bias toward expected items. In order to fully assess the possible contributions of primed popout and nonprimed sink-in to the observed difference in reportability between primed and nonprimed words in mixed arrays, it is necessary to establish baseline levels of reportability in unmixed arrays - that is, arrays containing either two primed words or two nonprimed words (see note 1). Without both baselines, it is impossible to tell whether the differential reportability is due to a perceptual advantage of the primed members of mixed arrays over and above the baseline advantage of primed words. Although Dark et al. established a nonprimed baseline by means of which they were able to show the suppression of the nonprimed words in the mixed arrays, they did not include unmixed primed arrays with which to assess the possible perceptual facilitation of the primed words. Thus, the design did not allow an assessment of the possible contribution of primed popout to the superior reportability of the primed members of mixed arrays.

Another feature of their paradigm is that primed words and their nonprimed companions were not equated in terms of associative value. Fowler, Wolford, Slade, and Tassinary (1981) observed a response bias toward words that are high associates of prime words. Indeed, Dark et al. (1996) observed a similar effect of associative value between words that were high associates of the primes and filler words even when these words occupied nonprimed arrays. However, their design did not permit them to completely disentangle the possible contributions to the superior reportability of primed words of a baseline advantage of primed over nonprimed words, the effect of associative value on perceptibility, and a separate, perceptual bias toward primed items.

Finally, the arrays used by Dark et al. (1996) consisted of just two locations, one above and the other below fixation. Although position did not interact with any variables of interest, report accuracy was much greater for the top word than for the bottom word, and this strong positional effect might have modulated the effects of associative priming on the spatial distribution of perceptual processing. These effects might be more robust and conspicuous if processing is more evenly distributed over larger arrays.

We conducted a partial replication of the Dark et al. (1996) research using procedures informed by all three of these methodological considerations. First, we included unmixed primed arrays in order to establish a baseline by which to measure primed popout. Second, the primed and nonprimed words in mixed (as well as unmixed) arrays were equated in terms of associative value, thereby obviating any effect of this variable on report accuracy. Finally, we attempted to reduce positional effects by increasing the spatial uncertainty of the two words in an array.

\section{METHOD}

Five compositions of two-word arrays were compared: one-primed arrays, in which only one of the words was related to the prime; bothprimed arrays, in which both words were related to the prime as well as to each other; both-nonprimed/unrelated arrays, in which the two words were related neither to the prime nor to each other; both-nonprimed/ related arrays, in which the two words were related to each other but not to the prime; and nonprimed filler arrays composed of items of relatively low associative value. Various comparisons between different array compositions in terms of report accuracy were of interest. A comparison of both-primed arrays with the primed members of one-primed arrays permitted an assessment of primed popout, a comparison of bothnonprimed/unrelated arrays with the nonprimed members of one-primed arrays permitted an assessment of nonprimed sink-in, a comparison of both-nonprimed/unrelated arrays with both-nonprimed/related arrays permitted an assessment of the possible facilitatory effect of interitem association in the absence of priming, and a comparison of the filler arrays with the both-nonprimed/unrelated arrays permitted a further assessment of the effect of associative value.

\section{Apparatus and Stimulus Material}

The experiment was conducted on an IBM-386-compatible computer with a VGA color monitor, Observers sat at a comfortable viewing distance of approximately $60 \mathrm{~cm}$ from the screen. The room was darkened so that most of the ambient light emanated from the monitor. Observers wore headphones over which a low level of white noise was presented to minimize distraction.

Words composing all except the filler arrays were drawn from the Connecticut Free Association Norms (Bousfield, Cohen, Whitmarsh, \& Kincaid, 1961). Altogether, 120 triplets of associatively related words were drawn. Each triplet consisted of a prime word and its two highest associates (e.g., BUNGALOW: COTTAGE, HUT; BEAR: CUB, BROWN), with the stipulations that each word had to be three to eight letters in length and that each associate had to have been generated by at least $10 \%$ of the 116 participants in the normative study. Triplets remained intact for the both-primed arrays but had to be broken up to create the one-primed, both-nonprimed/unrelated, and both-nonprimed/related arrays. Words from the triplets were randomly assigned to the different arrays. Filler arrays were composed of words drawn from the Kučera and Francis (1967) word norms, with the same length restrictions as those for the 
words composing the triplets. Because only a few of these filler words happened to be represented in the associative norms, it is reasonable to assume that they were of lower associative value than the words composing the triplets.

\section{Observers and Procedures}

Forty-five observers were recruited from undergraduate psychology classes at the University of Utah and participated in the experiment in return for course credit. All observers reported normal or corrected-tonormal vision and fluency in English. The experimental session lasted about 45 min and consisted of 16 trials of practice on filler arrays followed by 192 critical trials. The duration of array exposure was set at $1,000 \mathrm{msec}$ on the first practice trial but was gradually reduced to $200 \mathrm{msec}$ by the last practice trial. The critical trials constituted a random sequence of 24 one-primed trials, 24 both-primed trials, 24 bothnonprimed/unrelated trials, 24 both-nonprimed/related trials, and 96 filler trials. Three independent constructions of the experiment were created, and each one was assigned to a random third of the observers.

Each trial consisted of a one-word priming array followed by a twoword test array. Both prime and test words were presented in a $3 \times 3$ matrix in which the center cell contained an asterisk and served as a fixation point. Each cell subtended visual angles of about $2.4^{\circ}$ horizontally and about $1.3^{\circ}$ vertically. The matrix as a whole subtended about $7.2^{\circ}$ horizontally and about $3.9^{\circ}$ vertically.

The prime word was presented at random in one of the eight peripheral locations for $200 \mathrm{msec}$ and was followed by a 100 -msec mask formed by strings of $10 \mathrm{Xs}$ in all eight locations. A 1.5-sec interval followed the offset of the mask, during which time observers attempted to name aloud the word they had just seen. Subsequently, the prime word reappeared in the center of the screen. Observers were instructed to press "1" on the numerical keypad of their computer keyboard if they had correctly named the word and "4" if they had not. After the observer responded, a 200-msec interval preceded the onset of the test array. This method of presenting the prime was intended to ensure that the prime word was semantically processed, to maintain a task continuity across prime and the test arrays, and to obscure as much as possible the relationship between the prime and test arrays. The fact that the filler trials reduced the proportion of valid priming (viz., one-primed and bothprimed) trials to .25 should have helped to obscure this relationship.

Each test array was also exposed for $200 \mathrm{msec}$ and followed first by a 100 -msec mask and then by a 1.5 -sec interval during which the observer attempted to report the test words. The two test words were randomly located in the eight peripheral cells with the restriction that neither appear in the location that had been occupied by the preceding prime word. Following the report interval, observers received a selfscoring array consisting of the two test words, randomly numbered "(1)" and "(2)," and two other options, "(3) both" and "(4) neither." Observers were instructed to press the appropriate key, giving themselves credit only for reports that were exactly correct. The interval between the response to a test array and the onset of the prime array for the ensuing trial was $250 \mathrm{msec}$.

An experimenter in an adjacent room could overhear some of the observers' responses and kept a partial independent record of response accuracy. Subsequent comparisons of the experimenter's tallies with the self-scored records showed that they agreed over $95 \%$ of the time. Because of this high agreement and because the experimenter scoring was incomplete and error prone, all analyses were run on the self-scored data. Every 30 trials, the program paused for a self-paced rest period.

\section{RESULTS}

As was the case in the Dark et al. (1996) research, observers rarely reported more than one of the two words in an array. Just one word was correctly reported on $58 \%$ of the trials, and both words were correctly reported on only $3 \%$ of the trials. The majority of errors produced by observers were errors of omission. The primary unit of analysis was the probability of correct report for each ob-

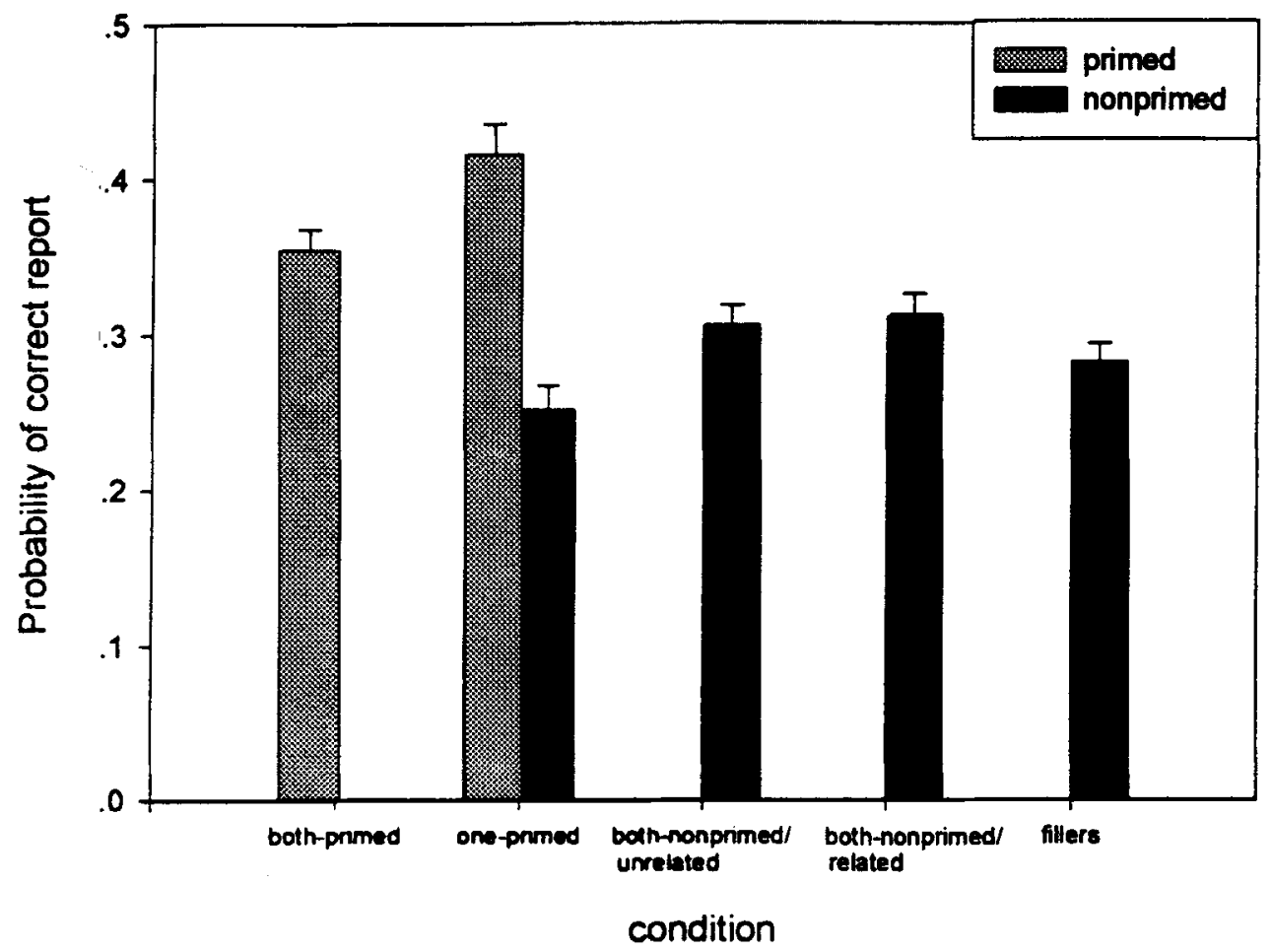

Figure 1. Probability of correct report in each of six conditions (the five array compositions and the two types of words nested in the one-primed arrays). Error bars indicate standard errors. 
server of the words in each of the five array compositions. These data are summarized in Figure 1. A one-way repeated measures analysis of variance (ANOVA) was used to compare all of the probabilities summarized in Figure 1 except those for the filler arrays. Because the probabilities were computed separately for primed and nonprimed members of the one-primed arrays, this analysis comprised five conditions, the remaining three conditions being both-primed, both-nonprimed/unrelated, and bothnonprimed/related. The five conditions differed reliably $\left[F(4,176)=28.06, M S_{\mathrm{e}}=.01, p<.01\right]$, and a NewmanKeuls analysis indicated that all of the probabilities differed reliably from one another except those for the bothnonprimed/unrelated and both-nonprimed/related arrays.

These findings replicate and extend the main findings of Dark et al. (1996). A word in a both-primed array was significantly more likely to be reported than a comparable word in a both-nonprimed array, a facilitatory main effect of priming comparable to the baseline effects of expectancy observed in other studies. The probability of reporting the nonprimed word in a one-primed array was below the all-nonprimed baseline, replicating the nonprimed inhibition reported by Dark et al. The probability of reporting the primed word in a one-primed array was higher than that for the both-primed baseline, indicating that the superior reportability of the primed word was due to both primed popout and nonprimed sink-in. Finally, the statistical equivalence of the both-nonprimed/ unrelated and both-nonprimed/related conditions indicates that report accuracy was not enhanced by an associative relationship between the two words in an array in the absence of priming. These effects were obtained even though the primed and nonprimed words were equated in terms of associative value, and even though our eight-cell arrays rendered the locations of the two words spatially unpredictable. ${ }^{2}$

A paired-comparison $t$ test revealed one other result of interest. Specifically, report accuracy was reliably lower for the filler arrays than for the both-nonprimed/unrelated arrays $\left[t(1,44)=2.64, M S_{\mathrm{e}}=0.009, p<.05\right]$. This difference is consistent with the effect of associative value reported by Fowler et al. (1981), although it was controlled for in our tests of priming and could not account for the observed popout and sink-in effects.

\section{DISCUSSION}

Having shown that the Dark et al. (1996) findings are replicable and based as much on primed popout as nonprimed sink-in, we are left with the empirical ambiguity posed by the juxtaposition of these findings with the novel and nonprimed popout and familiar and primed sink-in effects observed previously in our own laboratory (e.g., DeWitt, 1994; Johnston et al., 1990; Johnston et al., 1996). To account for this and other conflicting evidence concerning the effects of expectation, Johnston and Hawley (1994) developed a computational model, termed mismatch theory, in which the bias toward expected inputs operates at an upper, conceptual level of input processing and the bias toward unexpected inputs at a lower, physical level.

Some of the computational details of mismatch theory have been provided by Johnston and Hawley (1994). Input processing proceeds up- ward through two tiers of nodes: a lower tier representing the physical properties of the inputs and an upper tier representing the identities or conceptual properties. Nodes in the lower tier are connected by innate inhibitory links and nodes in the upper tier by acquired facilitatory links. The two tiers are themselves interconnected by both bottom-up facilitatory links and top-down inhibitory links. The latter links play a key role in rendering mismatch theory biased toward both expected and unexpected inputs. Because of an associative spreading of activation in the upper tier, expected inputs receive more conceptual processing than unexpected inputs. However, because the amount of top-down inhibition is proportional to the amount of conceptual activation, expected inputs receive less physical processing than unexpected inputs. Thus, conceptual processing is biased toward expected inputs and physical processing toward unexpected inputs.

Accordingly, whether the primed or the nonprimed words "pop out" from mixed arrays depends on the level of processing on which the tasks are most dependent and the measures most sensitive. The primed words should have the edge over nonprimed words on measures of conceptual processing, but the nonprimed words should have the edge on measures of physical processing. The fact that nonprimed popout has been observed in terms of localization accuracy and primed popout in terms of report accuracy may be attributed, at least in part, to the possible differential sensitivity of these measures to physical and conceptual processing, respectively. Localization tasks may be more sensitive to physical processing, whereas identification tasks may be more sensitive to conceptual processing. Similar opposing effects of expectancy on different tasks and measures have been observed by several others (e.g., Besner, Smith, \& MacLeod, 1990; von Hippel, Jonides, Hilton, \& Sowmya, 1993; for other examples, see Johnston \& Hawley, 1994).

Another factor contributing to the popout and sink-in effects observed in the present study may be response competition. A plausible assumption is that the likelihood of a correct report is based on both the absolute and relative amounts of conceptual processing of the two words in an array. The absolute levels of processing should be higher for both-primed than for both-nonprimed arrays, accounting for the baseline advantage of priming. In one-primed arrays, the primed members should have the advantage in terms of relative levels of conceptual processing as well as absolute levels. Inasmuch as the vast majority of correct reports were restricted to just one word per array, the words that were reported may be assumed to have been those that received more conceptual processing than their companions. This response competition should not systematically favor either word in a both-primed or bothnonprimed array, but should tend to favor the primed word in a oneprimed array. Accordingly, the primed word should tend to win the response competition in the one-primed arrays, causing its report to rise above the primed baseline and the report of the nonprimed loser to fall below the nonprimed baseline, contributing to the observed primed popout and nonprimed sink-in effects on report accuracy. This kind of response competition would not have been as much of a factor in our prior research on localization accuracy because on each trial in those studies observers were probed for just a single localization response.

We conclude by reiterating the primary theoretical motivation for the present research, namely the thesis developed by Johnston and Hawley (1994) that complex, dynamic systems like the mind/brain need to be biased simultaneously toward expected and unexpected inputs so that they can conform to the stable features of their environments and still remain sufficiently flexible to detect and accommodate changes. Both biases have now been demonstrated in our own laboratory: popout of unexpected inputs in our prior research and popout of expected inputs in the present study.

\section{REFERENCES}

Besner, D., Smith, M. C., \& MacLeod, C. M. (1990). Visual word recognition: A dissociation of lexical and semantic processing. Journal of Experimental Psychology: Learning, Memory, \& Cognition, 16, 862-869.

Bousfield, W. A., Cohen, B. H., Whitmarsh, G. A., \& Kincaid, W. D. (1961). The Connecticut Free Association Norms (Tech. Rep. No. 35). Storrs, CT: University of Connecticut. 
Dark, V. J., Vochatzer, K. G,. \& Van Voorhis, B. A. (1996.) Semantic and spatial components of selective attention. Journal of Experimental Psychology: Human Perception \& Performance, 22, 63-81

DEWITT, M. J. (1994). Attention capture by primed and unprimed stimuli. Unpublished doctoral dissertation, University of Utah.

Fowler, C. A., Wolford, G., SLADE, R., \& TASSinary, L. (1981). Lexical access with and without awareness. Journal of Experimental Psychology, General, 110, 341-362.

Johnston, W. A., \& HAWLEy, K. J. (1994), Perceptual inhibition of expected inputs: The key that opens closed minds. Psychonomic Bulletin \& Review, 1, 56-72.

Johnston, W. A., Hawley, K. J., Plewe, S. H., Elliott, J. M. G., \& DEWITT, M. J. (1990). Attention capture by novel stimuli. Journal of Experimental Psychology: General, 119, 397-411.

Johnston, W. A., \& Schwarting, I. S. (1996). Reassessing the evidence for novel popout. Journal of Experimental Psychology: General, $125,208-212$.

JOHNSTON, W. A., \& SCHWARTING, I. S. (1997). Novel popout: An enigma for conventional theories of attention. Journal of Experimental Psychology: Human Perception \& Performance, 25, 622-631.

Johnston, W. A., Schwarting, I. S., \& Hawley, K. J. (1996). Novel popout, perceptual inhibition, and the stability/plasticity dilemma. In A. F. Kramer, M. G. H Coles, \& G. D. Logan (Eds.), Converging operations in the study of visual selective attention (pp. 315-335). Washington, DC: American Psychological Association.

KuČERA, H., \& FranCIs, W. N. (1967). Computational analysis of present-day American English. Providence, RI: Brown University Press. von Hippel, W., Jonides, J., Hilton, J. L., \& Sowmya, N. (1993). The inhibitory effects of schematic processing on perceptual encoding. Journal of Personality \& Social Psychology, 64, 921-935.

\section{NOTES}

1. We define attention operationally in terms of differential levels of performance (e.g., localizability or reportability) either within or between arrays. Thus, the terms popout, sink-in, and attention capture refer only to the empirical effects and not to the subjective perceptual experiences of the observers. For example, an unexpected item in the company of expected items may be said to "pop out" or "capture" attention if it is more localizable or reportable either than its expected companions (within-array popout) or than it would be in the company of other unexpected items (between-array popout). Because within-array popout may be attributed to the usual baseline advantage of expected items in terms of perceptibility, it is necessary to include the betweenarray comparison in order to assess any selective processing of expected inputs over and above a baseline effect (e.g., perhaps indicating a concentration of "limited processing capacity" on the "attended" items).

2. The proportion of correct reports varied across locations from a low of .15 in the lower right location to a high of .67 in the upper middle location. In accordance with Dark et al. (1996), report accuracy was consistently higher in the upper locations than in the corresponding lower locations. However, the number of observations for each condition in each location was too low and variable to include location as a factor in the main analyses.

(Manuscript received July 5, 1996;

revision accepted for publication December $2,1997$. 